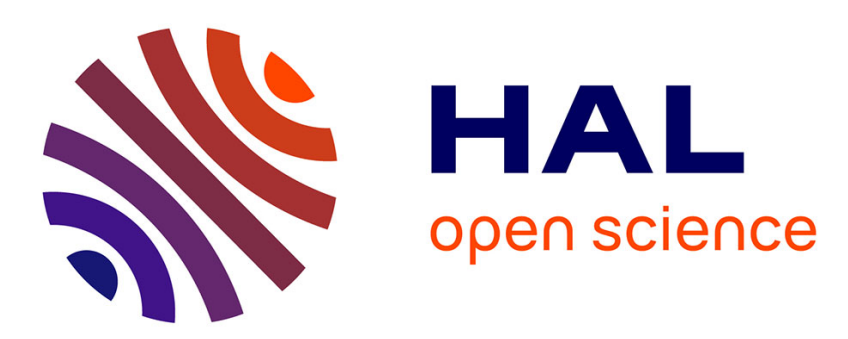

\title{
Distributed and backstepping boundary controls for port-Hamiltonian systems with symmetries
}

Ngoc Minh Trang Vu, Laurent Lefevre, Rémy Nouailletas

\section{To cite this version:}

Ngoc Minh Trang Vu, Laurent Lefevre, Rémy Nouailletas. Distributed and backstepping boundary controls for port-Hamiltonian systems with symmetries. Mathematical and Computer Modelling of Dynamical Systems, 2017, 23 (1), pp.55-76. 10.1080/13873954.2016.1232280 . hal-01623859

\section{HAL Id: hal-01623859 \\ https://hal.science/hal-01623859}

Submitted on 25 Oct 2017

HAL is a multi-disciplinary open access archive for the deposit and dissemination of scientific research documents, whether they are published or not. The documents may come from teaching and research institutions in France or abroad, or from public or private research centers.
L'archive ouverte pluridisciplinaire HAL, est destinée au dépôt et à la diffusion de documents scientifiques de niveau recherche, publiés ou non, émanant des établissements d'enseignement et de recherche français ou étrangers, des laboratoires publics ou privés. 


\title{
Distributed and backstepping boundary controls for port Hamiltonian systems with symmetries
}

\author{
Ngoc Minh Trang VU ${ }^{a}$, Laurent LEFÈVRE ${ }^{b \star}$ and Rémy NOUAILLETAS ${ }^{a}$ \\ (Received 00 Month 20XX; final version received 00 Month 20XX)
}

${ }^{a}$ CEA, IRFM, F-13108 Saint-Paul-Lez-Durance, France

${ }^{b}$ Univ. Grenoble Alpes, LCIS, F-26902, France

Email: ngoc-minh-trang.vu@cea.fr; laurent.lefevre@lcis.grenoble-inp.fr; remy.nouailletas@cea.fr

\begin{abstract}
A geometric spatial reduction for the port Hamiltonian models is presented in this paper. It is based on the projection which make use of the symmetries and on the preservation of the "natural" power pairing for the considered system. Thanks to this reduction, an Interconnection and Damping Assignment Passivity Based Control (IDA-PBC-like) synthesis for infinite dimensional port Hamiltonian systems (PHS) is investigated. As for the finite dimensional case, a feedback control transforms the original model into a closed-loop target Hamiltonian model. Both distributed control and boundary control are used. The finite rank distributed control is determined to solve an average IDA-PBC matching equation. A backstepping boundary control is used to stabilize the matching error. The control model chosen to illustrate the approach is the so-called resistive diffusion equation for the radial diffusion of the poloidal magnetic flux.

Keywords: distributed parameter systems, port-Hamiltonian systems, reduction, spatial symmetries, passivity based control, backstepping control, plasma control
\end{abstract}

\section{Introduction}

Infinite dimensional port-Hamiltonian systems (PHS) have recently become more and more popular either in the system theory community as a class of naturally well-posed (linear) systems (cf. [25]) or suitable for control designs based on Casimir Function (cf. $[10,15])$ and Control by Interconnection (CbI) or energy shaping (cf. $[7,9])$. On the other hand, Interconnection and Damping Assignment Passivity Based Control (IDA-PBC) design methods have been successful in the control of nonlinear (and linear) finite dimensional PHS in $[11,14]$. Roughly speaking, it makes use of the feedback control to match the original system with a desired system written in the form of a closed-loop asymptotically stable Hamiltonian system. The closed-loop system parameters, namely the desired closed-loop Hamiltonian, interconnection and damping matrices may be freely chosen in a large class of possible values, which is as the same time the most interesting advantage and one of the drawbacks of the method. Indeed, one has to solve a matching equation with some specific constraints for each of these parameters. Even in the finite dimensional case, finding a solution to the matching equation which satisfies these constraints still relies essentially on the physical intuition and the control experience of the designer.

In this paper, we address the IDA-PBC design problem for a class of distributed parameter systems. This class is made of those 3D systems of balance equations which may be reduced to 1D PHS by using spatial symmetries. We propose a 
reduction method which aims at reducing the 3D model while preserving its geometric interconnection structure. More precisely, the reduction relies on specific partial evaluations (integrals) for each kinds of effort and flow variables (differential forms) on some coordinate surfaces or contours, in order to reduce their dimensions and degree. The reduction is performed in such a way that the power pairing value between effort and flow variables remains unchanged, preserving in this way the geometric (Stokes-Dirac) interconnection structure of the original system and, consequently, many of its qualitative properties (such as passivity, losslessness symplecticity and stability, if any). This geometric nature of the reduction method is essential since our main motivation remains the derivation of 1D PHS model which allows the design of non linear control laws based on the model structure.

An IDA-PBC design for infinite dimensional PHS with both finite rank distributed control and boundary control is then proposed. The controls are designed in such a way that the matching error for the distributed control is compensated by the boundary control. More precisely, while keeping the geometrical interconnection structure (namely the Dirac structure) unchanged, we propose energy shaping and damping assignment to match a restricted class of closed-loop PHS. On one hand, because of the system constraints on the choice of the design parameters, it is hard to find a general solution to the matching equation. On the other hand, if the proposed controller does not satisfy exactly the matching equation and the constraints, there is no guaranteed stability for the desired closed-loop equilibrium.

In order to overcome this problem, we use the finite rank distributed control in the state equation and the boundary control simultaneously. We propose an approximated solution to the matching equation such that the matching error may be compensated by the boundary control via a "propagation function" (or backstepping control) to be determined. This backstepping control is inspired by the one developed in [6], to match reference diffusion or wave equations.

The whole methodology (structured reduction and model based control design) is then applied to the control of the poloidal magnetic flux radial profile in a tokamak (cf. [24]), the experimental facility used to create controlled nuclear fusion reactions. The objective of the control (regulation) is to achieve some non uniform steady state radial flux profiles which help to avoid magneto-hydrodynamic (MHD) instabilities and to improve the plasma confinement. It is one of the control challenges which is frequently studied in the recent tokamak control literature [1], [5], [13]. In [21], the IDA-PBC laws were derived for a finite-dimensional PHS model representing the plasma dynamics. In this paper, the infinite-dimensional PHS case is investigated. The reduction approach is applied to the $3 \mathrm{D}$ model derived from the mass, momentum, entropy and electromagnetic balance equations [19], assuming axisymmetry and quasi-static hydrodynamic equilibrium. These two assumptions are equivalent to assuming a toroidal symmetry. Indeed, in these cases, magnetic surfaces (which are also isothermal and isobaric) are nested tori whose index may be considered as the radial coordinate [3]. In this problem, both boundary (external magnetic coils) and distributed (antennas) actuators are available. A non-inductive current injection, generated by the antennas, plays the role of the finite rank distributed control, while the loop voltage generated by the transformer effect of the external coils realizes the boundary control. The distributed control is finite rank since only the total incoming power and the angle for the injected waves are controlled while the radial distribution (shape) of the control action is fixed for a given actuator (usually a Gaussian-like spatial distribution). Therefore we can't satisfy the matching equation (nor reach a given equilibrium profile) with the distributed actuator only and the use of the boundary control is justified. Contrarily to the 
usual boundary homogenization technique (where the boundary action is "lifted" from the boundary conditions to the state evolution equation), we define a boundary control which includes both the "usual" boundary action and a supplementary boundary control which will propagate inside the spatial domain to stabilizing the matching error.

The paper is organized as follows. In Section 2 we define the class of 1D PHS for which the design method applies. In Section 3, we define the geometric reduction method (from 3D to 1D) and apply it to the plasma model to obtain a model in the desired 1D class which is equivalent to the usual resistive diffusion equation. In Section 4, we recall the IDA-PBC design principle which is adapted to the infinite dimensional case and the exponential stability for the energy shaping and damping assignment design for the linear port Hamiltonian case. In Section 5, the coupled designs of a relaxed (average) IDA-PBC distributed control and of a stabilizing backstepping boundary control are proposed. In Section 6, the design method is applied to the 1D resistive diffusion control problem. Simulation results obtained from RAPTOR code (which is used for real time control of the TCV (CRPP, EPFL) and Tore Supra (CEA Cadarache) tokamaks) are investigated. The papers ends with a summary of the results presented in Section 7 .

\section{1D port Hamiltonian systems with finite rank distributed control and boundary control}

We will investigate the control problem for a class of distributed parameter PHS (cf. $[15,17]$ ) with both boundary and distributed finite rank controls defined as:

$$
\begin{cases}\dot{x}(t) & =[\mathcal{J}-\mathcal{R}] \delta_{x} \mathrm{H}+g u_{1}(t) \\ \left\langle u_{2}(t), y_{2}(t)\right\rangle & =\mathcal{B}_{\mathcal{J}}\left(\delta_{x} \mathrm{H}, \delta_{x} \mathrm{H}\right)\end{cases}
$$

where $x(t) \in \mathcal{X}=L^{2}\left(\mathcal{Z}, \mathrm{R}^{n}\right), \forall t \geq 0$, denoting $\mathcal{Z}=[a, b]$, the 1D spatial domain. The distributed control action has rank one ${ }^{1}$ and $u_{1}$ is a time dependent scalar signal $u_{1}(t) \in \mathrm{R}, \forall t \geq 0$ while the control map $g \in L^{2}\left(\mathcal{Z}, \mathrm{R}^{n}\right)$ represents the spatial distribution of this control action. The differential operator $\mathcal{J}$ is assumed to be formally skew-symmetric, i.e. $\mathcal{J}=-\mathcal{J}^{*}$ where $\mathcal{J}^{*}$ denotes the formal adjoint of $\mathcal{J}$ with respect to the usual inner product in $L^{2}\left(\mathcal{Z}, \mathrm{R}^{n}\right)$ (cf. [8] Corollary 3.3). For the sake of simplicity we will consider only the restricted class of differential operators of the form $\mathcal{J}=P_{1} \partial_{z}+P_{0}$, with domain $D(\mathcal{J})=H^{1}\left(\mathcal{Z}, \mathrm{R}^{n}\right)$, where $P_{1}=P_{1}^{T}$ is a non singular square symmetric matrix of order $n$ and $P_{0}=-P_{0}^{T}$ is a skew-symmetric one. Note that this class may be generalized to systems with higher order spatial derivatives such as in $[18,25]$. The dissipation is defined using a non negative self-adjoint operator $\mathcal{R}$. In the sequel we will consider a simple multiplicative operator with a positive semidefinite matrix in $L^{2}\left(\mathcal{Z}, \mathcal{M}_{n}(\mathrm{R})\right)$ where $\mathcal{M}_{n}(\mathrm{R})$ is the set of real square matrices of order $n$. The total energy stored in the system, i.e. the Hamiltonian function $\mathrm{H}$, is computed using a smooth Hamiltonian density $\mathcal{H}: \mathcal{X} \rightarrow \mathrm{R}$ according to:

$$
\mathrm{H}=\int_{\mathcal{Z}} \mathcal{H}
$$

\footnotetext{
${ }^{1}$ although extension to the general finite rank case is straightforward, it is not necessary for the application case presented in this paper
} 
The Hamiltonian density $\mathcal{H}$ is assumed to be regular enough to define properly the variational derivative $\delta_{x} \mathrm{H}(x)$ with respect to $x$ such that [17]:

$$
\mathrm{H}(x+\epsilon \delta x)=\mathrm{H}(x)+\epsilon \int_{\mathcal{Z}}\left\langle\delta_{x} \mathrm{H}(x), \delta x\right\rangle+O\left(\epsilon^{2}\right), \forall x, \delta x \in H^{1}\left(\mathcal{Z}, \mathrm{R}^{n}\right) ; \forall \epsilon>0
$$

where $\langle\cdot, \cdot\rangle$ denotes the usual inner product in $\mathrm{R}^{n}$.

The bilinear boundary operator $\mathcal{B}$ with domain $H^{1}\left([a, b], \mathrm{R}^{n}\right)$ is defined as the differential operator induced on the boundary $\partial \mathcal{Z}=\{a, b\}$ by the differential operator $\mathcal{J}$ in the sense of definition II.1 (see also lemma 3.1 in [8]).

Definition II.1: Consider a linear differential operator $L: H^{1}\left(\mathcal{Z}, \mathrm{R}^{n}\right) \rightarrow$ $L^{2}\left(\mathcal{Z}, \mathrm{R}^{n}\right)$ and denote $L^{*}$ its formal adjoint. Then there exists a unique bilinear differential operator $\mathcal{B}_{L}: H^{1}\left(\mathcal{Z}, \mathrm{R}^{n}\right) \times H^{1}\left(\mathcal{Z}, \mathrm{R}^{n}\right) \rightarrow L^{1}(\partial \mathcal{Z}, \mathrm{R})$ such that:

$$
\int_{\mathcal{Z}}\left(\langle h, L f\rangle-\left\langle L^{*} h, f\right\rangle\right)=\int_{\partial \mathcal{Z}} \mathcal{B}_{L}(f, h), \forall f, h \in H^{1}\left([a, b], \mathrm{R}^{n}\right)
$$

We say that $\mathcal{B}_{L}$ is the differential operator induced on the boundary $\partial \mathcal{Z}$ by the differential operator $L$.

For instance, if we consider the first order spatial derivative operator

$$
L=\left(\begin{array}{ll}
0 & 1 \\
1 & 0
\end{array}\right) \partial_{z}
$$

together with the spatial domain $\mathcal{Z}=[a, b]$, then for all $f, h \in H^{1}\left([a, b], \mathrm{R}^{2}\right)$ :

$$
\begin{aligned}
\int_{\mathcal{Z}}\left(\langle h, L f\rangle-\left\langle L^{*} h, f\right\rangle\right) & =\int_{\mathcal{Z}} \partial_{z}\left(h_{2} f_{1}+h_{1} f_{2}\right) \mathrm{d} z \\
& =\int_{\partial \mathcal{Z}}\left(h_{2} f_{1}+h_{1} f_{2}\right)=\left.\left(h_{2} f_{1}+h_{1} f_{2}\right)\right|_{a} ^{b}
\end{aligned}
$$

Therefore the boundary operator $\mathcal{B}_{L}$ induced by the differential operator $L$ is the evaluation of $\left(h_{2} f_{1}+h_{1} f_{2}\right)$ on the boundary $\partial \mathcal{Z}=\{a, b\}$.

Such induced boundary operators may be constructed for a larger class of higherorder skew symmetric differential operators. The resulting class of systems of the form (II.1) together with the class of induced input-output variables which are admissible in order to define a well-posed linear problem and to generate a contraction semigroup are defined in [18]. The reader can refer to [8] for more details.

In the control system (II.1), the pair of boundary input and output port variables $u_{2}(t)$ and $y_{2}(t) \in \mathrm{R}^{n}$ are chosen to be conjugated in the sense that: $<u_{2}(t), y_{2}(t)>=\mathcal{B}_{\mathcal{J}}\left(\delta_{x} \mathrm{H}(x), \delta_{x} \mathrm{H}(x)\right)$. When there is no dissipation or distributed control action (source term) the energy balance equation reads:

$$
\frac{d \mathrm{H}}{d t}=\int_{\mathcal{Z}}\left\langle\delta_{x} \mathrm{H}(x), \dot{x}\right\rangle=\int_{\partial \mathcal{Z}} \mathcal{B}_{L}\left(\delta_{x} \mathrm{H}(x), \delta_{x} \mathrm{H}(x)\right)=\left\langle u_{2}, y_{2}\right\rangle
$$

In the example considered in this paper (see Section III.2), there is only one boundary point in $\partial \mathcal{Z}$ which corresponds to the external border of a toroidal plasma. This does not prevent us from defining the induced bilinear operator according to 
definition II.1 and the conjugated input-output pair of boundary port variables accordingly.

It must be noticed that the class of systems of the form (II.1) includes most classical hyperbolic examples such as wave, membrane, plates and beams equations, the shallow water equation, the Boussinesq, Korteg de Vries and Navier-Stokes flow equations, the Maxwell field equations, etc. but also some parabolic examples as it will be shown hereafter with the plasma poloidal flux resistive diffusion equation.

\section{Geometric reduction based on spatial symmetries and the resistive diffusion example}

A tokamak is a facility constructed with the shape of a torus (or dough-nut) in which a plasma is magnetically confined and heated in order to produce nuclear fusion reactions (see the Wesson's classical monograph [24]). The fusion research led by the ITER (International Thermonuclear Experimental Reactor) project needs, besides a better understanding of involved physical phenomena, a better optimization/control of the multi-domain couplings in order to improve the plasma confinement and to prove the feasibility of energy production. Control problems of tokamak plasma aim at many different objectives, including to handle MHD (magneto-hydrodynamic) instabilities and to control the current, temperature and pressure density profiles.

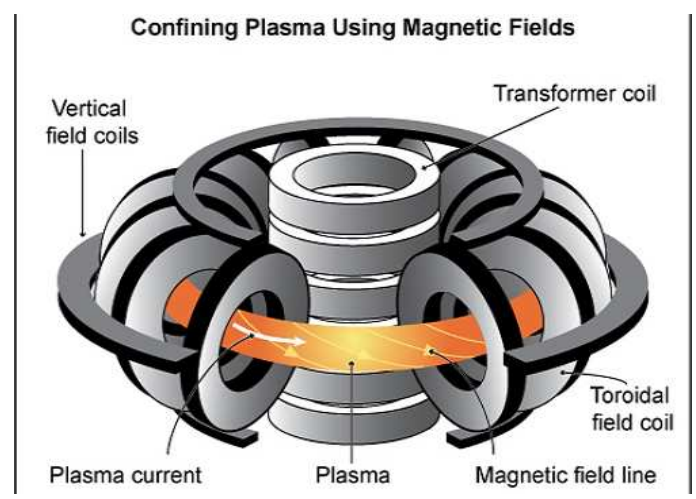

Figure 1. Simple scheme of Tokamak plasma

Tokamak plasma dynamics may be described using the port-Hamiltonian formulation. The mass, entropy, momentum and electromagnetic balance equations were first introduced in [20] in order to produce such a port Hamiltonian model for the electromagnetic domain and then developed in $[19,23]$ for the full thermomagneto-hydrodynamics model for the plasma. Classical axi-symmetry and quasistatic equilibrium assumptions (see [24]) may be used together with a symplectic reduction method to obtain a reduced $1 \mathrm{D}$ Tokamak control model which preserves not only the energetic properties but also the port-Hamiltonian structure (namely a Stokes-Dirac structure) from the original system. We present hereafter this spatial reduction which aims at transforming the $3 \mathrm{D}$ model into a $1 \mathrm{D}$ control model. It is mainly based on differential form evaluation in coordinates suitable for the assumed spatial symmetries and on power pairing conservation. We apply the ideas on the example of the plasma electromagnetic dynamics to obtain a model equivalent to the so-called 1D resistive diffusion equation. 


\section{III.1. Magnetic toric coordinates and the reduction principle}

In the tokamak example, the reduction and the spatial symmetries are based on two assumptions ${ }^{2}$ : axial symmetry with respect to the main torus axis and quasistatic equilibrium for the plasma (cf. [3, Chap.6]). With these two assumptions, it may be proved that the magnetic flux surfaces form a set of nested toroids which are simultaneously isobaric, isothermal and iso flux (see Figure 2.a and [24, sec. 3.2]). After a continuous mapping, these surfaces may be matched into nested regular toroidal surfaces with circular cross-sections and a set of magnetic toric coordinates $(\rho, \theta, \phi)$ (see Figure 2.b) may be defined such that $\rho$ denotes the index of the considered magnetic surface (the new "radial" coordinate) and such that all the system variables are independent of $\theta$ and $\phi$. Therefore the model may be projected onto a $1 \mathrm{D}$ domain $\mathcal{Z}=[0, a] \ni \rho$.

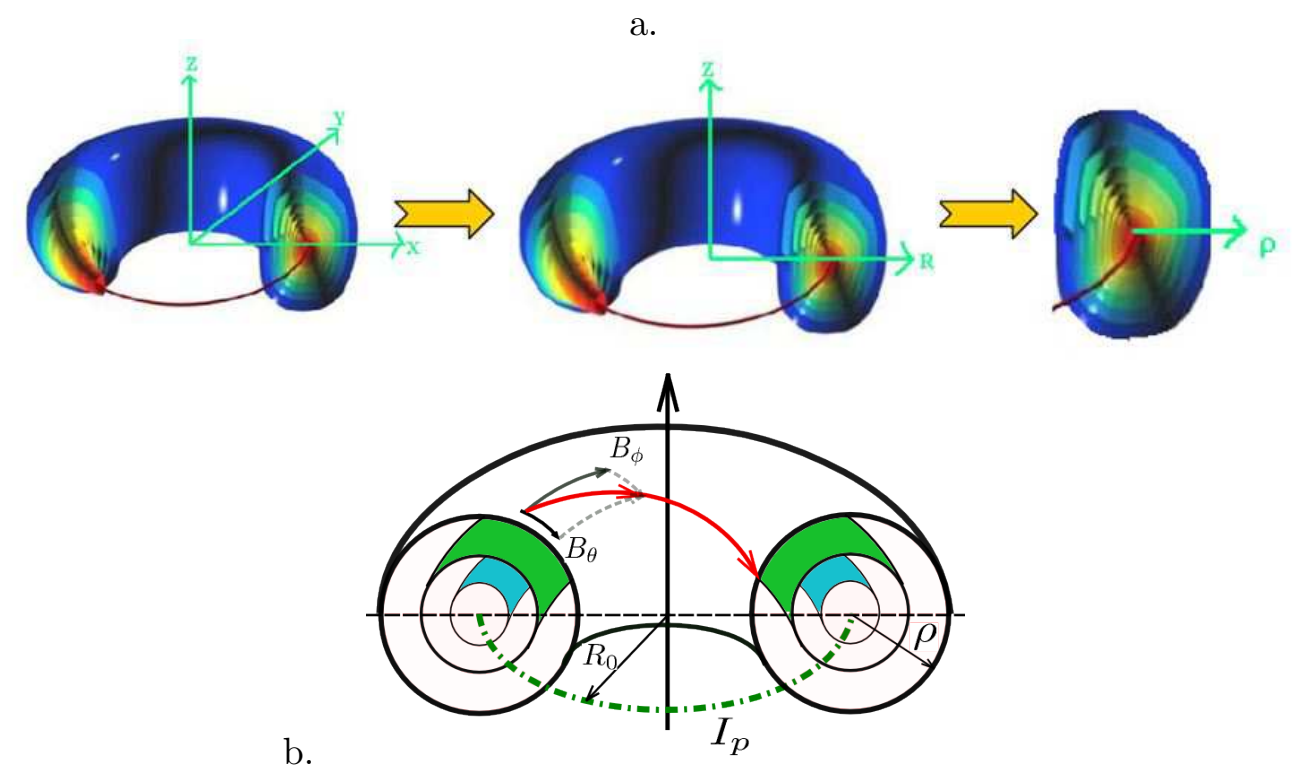

Figure 2. Magnetic toric coordinate: $\rho$ denotes the magnetic surface index (corresponding to the small radius $r), \theta$ the polar angle and $\phi$ the azimuth angle. $R_{0}$ denotes the principal radius of the plasma, $I_{p}$ the total plasma current and $B_{\theta}$ and $B_{\phi}$ the two components of the magnetic field

As illustrated in Figure 2.a, the magnetic surfaces cross-sections are usually not nested regular disks. Therefore the magnetic toric coordinates $(\rho, \theta, \phi)$ used in Figure 2.b are obtained from the "usual" or "real" geometric toric coordinates $(r, \theta, \phi)$ in 2.a. with a parametric description $r=r(\rho, \theta)$ of the magnetic surfaces cross-sections which is smooth for usual plasma shapes. Let $g_{\rho}, g_{\theta}, g_{\phi}$,

$$
\left\{\begin{array}{l}
g_{\rho}=\left(\partial_{\rho} r\right)^{2} \\
g_{\theta}=\left(\partial_{\theta} r\right)^{2}+r^{2} \\
g_{\phi}=\left(R_{0}+r \cos \theta\right)^{2}
\end{array}\right.
$$

denote the transformation coefficients between geometric toric coordinates $(r, \theta, \phi)$ and magnetic toric coordinates $(\rho, \theta, \phi)$. Then the volume element is $d V=$

\footnotetext{
${ }^{2}$ The very same reduction ideas would apply to other systems of balance equations with spatial symmetries such as for instance cylindrical or spherical geometries. The tokamak example and its particular toric geometry serves as an example to illustrate the power balance computations and the differential forms evaluations.
} 
$\sqrt{g} d \rho d \theta d \phi$ with $g=g_{\rho} g_{\theta} g_{\phi}$. For instance, the integration of energy density in magnetic toric coordinate reads:

$$
\mathrm{H}=\int_{V} \alpha \beta d V
$$

where $(\alpha, \beta)$ stands for the 1D energy variable pair such as $(B, H)$ in magnetic domain or $(D, E)$ in electric domain. This physical quantity $\mathrm{H}$ will be preserved via the spatial reduction into a $1 \mathrm{D}$ model defined on magnetic surfaces.

$$
\mathrm{H}=\int_{V} \alpha \beta d V=\int_{\mathcal{Z}} \bar{\alpha} \bar{\beta} d \rho
$$

where the $1 \mathrm{D}$ domain is denoted $\mathcal{Z}=[0, a] \ni \rho, a=\rho_{\max }$. The key feature is to determine the $1 \mathrm{D}$ variables $(\bar{\alpha}, \bar{\beta}) \in H^{1}\left(\mathcal{Z}, \mathrm{R}^{2}\right)$ in equation (III.2). We propose in this work to consider these reduced variables to be the value of the integrals of $\alpha$ and $\beta$ over some coordinate domains defined by the symmetries. The method will be illustrated and commented for the resistive diffusion example hereafter.

\section{III.2. From $3 D$ to $1 D$ port Hamiltonian formulation for the plasma dynamics}

The plasma electromagnetic 3D model was developed in port-Hamiltonian form in [20]. It is defined using the following Maxwell's equations:

$$
\left(\begin{array}{l}
-\partial_{t} D \\
-\partial_{t} B
\end{array}\right)=\left(\begin{array}{cc}
0 & -\nabla \times \\
\nabla \times & 0
\end{array}\right)\left(\begin{array}{l}
E \\
H
\end{array}\right)+\left(\begin{array}{l}
1 \\
0
\end{array}\right) J
$$

where all the variables are defined in the $3 \mathrm{D}$ domain. $E, H$ are the electric and magnetic field intensities, $D, B, J$ are the field flows and the total current density respectively, and the Nabla notation $\nabla \times$ stands for the curl operator (written in the three dimensional coordinate system $(\rho, \theta, \phi)$. The electromagnetic energy function (Hamiltonian) is:

$$
\mathrm{H}_{E M}=\frac{1}{2} \int_{V}(E D+H B) d V
$$

Let us now apply the geometric reduction described in the previous subsection to the magnetic domain to determine the corresponding $1 \mathrm{D}$ variables which are in $\mathcal{Z}$ 
domain:

$$
\begin{aligned}
\mathrm{H}(B) & =\frac{1}{2} \int_{V} H B d V \\
& =\frac{1}{2} \int_{V}\left(H_{\rho} B_{\rho}+H_{\theta} B_{\theta}+H_{\phi} B_{\phi}\right) \sqrt{g} d \rho d \theta d \phi \\
& =\frac{1}{2} \int_{\mathcal{Z}} d \rho\left[\int_{0}^{2 \pi}\left(\sqrt{g_{\theta}} H_{\theta}\right) d \theta \int_{0}^{2 \pi}\left(\sqrt{g_{\rho} g_{\phi}} B_{\theta}\right) d \phi\right. \\
& \left.+\int_{0}^{2 \pi}\left(\sqrt{g_{\phi}} H_{\phi}\right) d \phi \int_{0}^{2 \pi}\left(\sqrt{g_{\rho} g_{\theta}} B_{\phi}\right) d \theta\right] \\
& =\frac{1}{2} \int_{\mathcal{Z}} d \rho\left[\left(\bar{H}_{\theta}\right)\left(\bar{B}_{\theta}\right)+\left(\bar{H}_{\phi}\right)\left(\bar{B}_{\phi}\right)\right] \\
& =\frac{1}{2} \int_{\mathcal{Z}} \bar{H} \bar{B} d \rho
\end{aligned}
$$

where

$$
\left\{\begin{array}{lll}
\bar{H}_{\theta}=\int_{0}^{2 \pi} H_{\theta} \sqrt{g_{\theta}} \mathrm{d} \theta & \bar{H}_{\phi}=\int_{0}^{2 \pi} H_{\phi} \sqrt{g_{\phi}} \mathrm{d} \phi \\
\bar{B}_{\theta}=\int_{0}^{2 \pi} B_{\theta} \sqrt{g_{\rho} g_{\phi}} \mathrm{d} \phi & \bar{B}_{\phi}=\int_{0}^{2 \pi} B_{\phi} \sqrt{g_{\rho} g_{\theta}} \mathrm{d} \theta
\end{array}\right.
$$

Note that $H_{\rho}$ and $B_{\rho}$ vanish on the magnetic surfaces. The $1 \mathrm{D}$ reduced variables $(\bar{H}, \bar{B})$ correspond to the integral values of the corresponding $3 \mathrm{D}$ variables over a specific integral (coordinate) domain. In other words, the magnetic field intensity $H$ is integrated over a coordinate curl (depending on $\theta$ or $\phi$ ) while the magnetic field flow $B$ is integrated over a coordinate section. The same reduction is applied to the electric domain with the energy density:

$$
\mathrm{H}(D)=\frac{1}{2} \int_{V} E D \mathrm{~d} V=\frac{1}{2} \int_{\mathcal{Z}} \bar{E} \bar{D} d \rho
$$

and leads to the definition of the reduced variables $\bar{E}, \bar{D}$ as well as $\bar{J}$ :

$$
\begin{cases}\bar{E}_{\theta}=\int_{0}^{2 \pi} E_{\theta} \sqrt{g_{\theta}} d \theta & \bar{E}_{\phi}=\int_{0}^{2 \pi} E_{\phi \sqrt{g_{\phi}}} \mathrm{d} \phi \\ \bar{D}_{\theta}=\int_{0}^{2 \pi} D_{\theta} \sqrt{g_{\rho} g_{\phi}} \mathrm{d} \phi & \bar{D}_{\phi}=\int_{0}^{2 \pi} D_{\phi} \sqrt{g_{\rho} g_{\theta}} \mathrm{d} \theta \\ \bar{J}_{\theta}=\int_{0}^{2 \pi} J_{\theta} \sqrt{g_{\rho} g_{\phi}} \mathrm{d} \phi & \bar{J}_{\phi}=\int_{0}^{2 \pi} J_{\phi} \sqrt{g_{\rho} g_{\theta}} \mathrm{d} \theta\end{cases}
$$

The 3D model (III.3) thus transforms into a 1D model with a similar power pairing product. The Maxwell's equations in $\mathcal{Z}$ domain are simply written:

$$
\partial_{t}\left(\begin{array}{l}
-\bar{D} \\
-\bar{B}
\end{array}\right)=\left[\begin{array}{cc}
0 & -\mathrm{d}_{\mathcal{Z}} \\
\mathrm{d}_{\mathcal{Z}} & 0
\end{array}\right]\left(\begin{array}{l}
\bar{E} \\
\bar{H}
\end{array}\right)+\left(\begin{array}{l}
1 \\
0
\end{array}\right) \bar{J}
$$

The reduced model structure in (III.6) is kept in the same form as in the 3D model (III.3). The derivative $\nabla \times$ (curl operator) in $3 \mathrm{D}$ domain becomes $\mathrm{d} \mathcal{Z}=\left(\begin{array}{cc}0 & -1 \\ 1 & 0\end{array}\right) \partial_{z}$ in the $1 \mathrm{D}$ reduced spatial domain $\mathcal{Z}=[0,1]$ (the spatial variable will be normalized as $\left.z=\frac{\rho}{\rho_{\max }}\right)$.

We will be interested, from now, only the half of the model III.6 (describing all the components) which gives $\bar{B}_{\theta}$ and, $\bar{D}_{\phi}$ necessary to compute the magnetic 
poloidal flux:

$$
\left\{\begin{aligned}
\partial_{t} \underbrace{\left(\begin{array}{c}
\bar{D}_{\phi} \\
\bar{B}_{\theta}
\end{array}\right)}_{x}= & {[\underbrace{\left(\begin{array}{ll}
0 & 1 \\
1 & 0
\end{array}\right) \partial_{z}}_{\mathcal{J}}-\underbrace{\left(\begin{array}{cc}
\frac{C_{3(z)}}{\eta(z)} & 0 \\
0 & 0
\end{array}\right)}_{\mathcal{R}} \underbrace{\left(\begin{array}{cc}
\frac{1}{\epsilon C_{3(z)}} & 0 \\
0 & \frac{C_{2(z)}}{\mu}
\end{array}\right)\left(\begin{array}{c}
\bar{D}_{\phi} \\
\bar{B}_{\theta}
\end{array}\right)}_{\mathcal{Q} x}} \\
& +\left(\begin{array}{c}
-\bar{J}_{\text {ext }}-\bar{J}_{b s} \\
0
\end{array}\right) \\
\mathcal{B}\left(\delta_{x} \mathrm{H}, \delta_{x} \mathrm{H}\right) & =\left.\left(\begin{array}{c}
f_{\partial} \\
e_{\partial}
\end{array}\right)\right|_{z=1}=\left(\begin{array}{c}
V_{\text {loop }} \\
I_{p}
\end{array}\right) \\
\mathrm{H} & =\int_{\mathcal{Z}} \frac{1}{2} x^{T} \mathcal{Q} x
\end{aligned}\right.
$$

In this last equation, the total current density $\bar{J}_{\phi}(z)=\bar{J}_{\text {ext }}(z)+\bar{J}_{b s}(z)$ includes the internal bootstrap current $\bar{J}_{b s}(z)$ (a current source from multidomain couplings in plasma physics) and the external current source $\bar{J}_{\text {ext }}(z, t)=$ $f_{\text {ext }}(z) P_{\text {ext }}(t)$, whose spatial shape function is $f_{\text {ext }}(z)$, and external current power is $P_{\text {ext }}(t)^{3} . C_{2}=\frac{\sqrt{g_{\theta}}}{\sqrt{g_{\rho} g_{\phi}}}, C_{3}=\frac{\sqrt{g_{\rho} g_{\theta}}}{\sqrt{g_{\phi}}}$ are the toric coordinate coefficients. The plasma resistivity $\eta(z, t)$ is normally a parameter varying significantly with the plasma temperature. However, since the Thermo-Magneto-HydroDynamic couplings are not considered in this work, the plasma resistivity is assumed to be a given spatially non-uniform time varying parameter $\eta(z, t)$. The parameters $\epsilon$ and $\mu$ are the void permittivity and void permeability (since the actual tokamaks are operating at very low densities). The boundary power at the plasma edge $\left.f_{\partial} e_{\partial}\right|_{z=1}=I_{p} V_{\text {loop }}$ defines the boundary variables: $V_{\text {loop }}$ is the scalar loop voltage produced by the external electric coils, and $I_{p}$ is the total plasma current. There is no energy source at the center $\left.f_{\partial} e_{\partial}\right|_{z=0}=0$.

Remark III.1: Usually when dealing with the poloidal magnetic flux profile control problem, the electric current displacement $\partial_{t}\left(-\bar{D}_{\phi}\right)$ is neglected and the hyperbolic system (III.7) reduces to the parabolic resistive diffusion equation

Remark III.2: The distributed control action is finite rank since only the total power $P_{\text {ext }}$ and not its spatial distribution may be chosen. Therefore only two scalar input signals $\left(P_{\text {ext }}(t), V_{\text {loop }}(t)\right)$ may be used to regulate the whole profile of the magnetic field. In that sense, we may speak about an "finite-rank distributed" system).

To deal with the non-linearity, a feedforward control may be designed to reach the desired equilibrium profile [21]. Then the system is linearized and a feedback control is added to stabilize the linearization error. The port Hamiltonian formulation for the linearization of the resistive diffusion may be turned into the usual PHS form:

$$
\left\{\begin{array}{l}
\partial_{t}\left(\begin{array}{l}
x_{1} \\
x_{2}
\end{array}\right)=[\mathcal{J}-\mathcal{R}] \mathcal{Q}\left(\begin{array}{l}
x_{1} \\
x_{2}
\end{array}\right)+g u_{1} \\
\left(\begin{array}{l}
u_{2} \\
y_{2}
\end{array}\right)=\left.\mathcal{Q}\left(\begin{array}{l}
x_{1} \\
x_{2}
\end{array}\right)\right|_{z=1}
\end{array}\right.
$$

where $\left(x_{1}, x_{2}\right)^{T}$ and $\left(u_{1}, u_{2}\right)^{T}$ respectively denote the system errors $\delta\left(\bar{D}_{\phi}, \bar{B}_{\theta}\right)^{T}$

\footnotetext{
${ }^{3}$ The external current source power $P_{\text {ext }}(t)=P_{A}(t)-P_{B}(t)$ is in fact the power difference between the co-current $P_{A}(t)$ and counter-current $P_{B}(t)$ sources.
} 
and the feedback control $\delta\left(P_{\text {ext }}, V_{\text {loop }}\right)^{T}$ of the resistive diffusion system in (III.7), while $g=\left(-f_{\text {ext }}(z), 0\right)^{T}$ is the control mapping, and the boundary condition is satisfied:

$$
\begin{cases}\left.x_{1}\right|_{z=0}=\left.x_{2}\right|_{z=0} & =0 \\ \left.g\right|_{z=0} & =0\end{cases}
$$

Remark III.3: This port-Hamiltonian model in (III.8) indicates the error evolution of the origin system (III.7). The bootstrap current $\bar{J}_{b s}$, which is usually not a control signal, is taken into account in the feedforward computation.

Remark III.4: The system dissipation $\mathcal{R} \geq 0$ is not strictly positive definite but the closed-loop dissipation may be modified by using the distributed control $g u_{1}$ derived in (V.1). The boundary control $u_{2}$ in (V.5) then corrects the matching error and guarantees the stability of the closed-loop system.

Very often some safety factor ${ }^{4} q\left(\sim 1 / \bar{B}_{\theta}\right)$ profile is desirable and considered as the control objective for regulation. However, safety factor profiles are in one to one correspondence with magnetic field profiles $\bar{B}_{\theta}$.

\section{IDA-PBC design for 1D port Hamiltonian systems}

In this section, the IDA-PBC principle [12] will be revisited and adapted for a class of infinite dimensional PHS. The studied system class is defined with Hamiltonian function in quadratic form, which allows to prove the Lyapunov stabilization of the closed-loop (target) system.

\section{IV.1. The IDA-PBC principle}

The purpose of the IDA-PBC feedback control design, for finite dimensional PHS, is to match a target (or "desired") passive PHS with desired closed-loop interconnection structure, Hamiltonian function and damping. We will keep the same approach for infinite dimensional PHS and design a feedback control to transform the original system into the following closed-loop system:

$$
\begin{cases}\dot{x} & =\left[\mathcal{J}_{d}(x, t)-\mathcal{R}_{d}(x, t)\right] \delta_{x} \mathrm{H}_{d} \\ \left\langle\tilde{u}_{2}, \tilde{y}_{2}\right\rangle & =\mathcal{B}_{d}\left(\delta_{x} \mathrm{H}_{d}, \delta_{x} \mathrm{H}_{d}\right)\end{cases}
$$

where $\mathcal{J}_{d}=-\mathcal{J}_{d}^{*}$, a skew-symmetric operator with domain $D(\mathcal{J})=H^{1}\left(\mathcal{Z}, \mathrm{R}^{n}\right)$, and $\mathcal{R}_{d}=\mathcal{R}_{d}^{T} \in L^{2}\left(\mathcal{Z}, \mathcal{M}_{n}(\mathrm{R})\right)$, a non negative self-adjoint multiplicative operator, denote respectively the desired closed-loop system interconnection and damping operators. The desired closed-loop Hamiltonian $\mathrm{H}_{d}$ has the minimum at the desired equilibrium $x_{d}$. That means the first and second order variational derivatives of $\mathrm{H}_{d}$ must satisfy:

$$
\begin{cases}\delta_{x} \mathrm{H}_{d}\left(x_{d}\right) & =0 \\ \nabla^{2} \mathrm{H}_{d}\left(x_{d}\right) & >0\end{cases}
$$

\footnotetext{
${ }^{4}$ The safety factor $q$ is an important parameter for the system stability study, see [24]. It is equivalent to inverse of the plasma current profile.
} 
where $\nabla^{2} \mathrm{H}_{d}:=\nabla^{2} \mathrm{H}_{d}\left(x_{d}\right)$ is a bounded linear map in $H^{1}\left([a, b], \mathrm{R}^{n}\right)$ which is defined as the second order variational derivative, i.e. such that:

$\mathrm{H}_{d}\left(x_{d}+\epsilon \delta x\right)=\mathrm{H}_{d}\left(x_{d}\right)+\frac{\epsilon^{2}}{2} \int_{\mathcal{Z}}\left\langle\nabla^{2} \mathrm{H}_{d} \delta x, \delta x\right\rangle+O\left(\epsilon^{3}\right), \forall \delta x \in H^{1}\left(\mathcal{Z}, \mathrm{R}^{n}\right) ; \forall \epsilon>0$

since the first order variational derivative $\delta_{x} \mathrm{H}_{d}$ is zero.

Remark IV.1: In the infinite dimensional case, conditions (IV.2) are in general not sufficient to guarantee asymptotic stability. Whenever this asymptotic stability is defined (in the Lyapunov sense) with respect to some norm $\|\cdot\|$ defined on $\mathcal{X}:=L_{2}\left(\mathcal{Z}, \mathrm{R}^{n}\right)$, Arnold's first stability theorem for non linear systems guarantees the asymptotic stability if, besides the conditions (IV.2), $\nabla^{2} \mathrm{H}_{d}$ remains bounded and coercive with the chosen norm in $\mathcal{X}$ (see, e.g. [16]). This may be guaranteed by choosing the norm associated with the energy function $\mathrm{H}_{d}$ and a coercivity condition on the Hamiltonian function.

The desired closed-loop boundary operator $\mathcal{B}_{d}$ is the operator induced by the differential operator $\mathcal{J}_{d}$ which ensures that the couple $\left(\tilde{y}_{2}, \tilde{u}_{2}\right)$ is a passive inputoutput pair for the target system with respect to the storage functional $\mathrm{H}_{d}$. The definition and parameterization of all admissible passive input-output pairs which lead to a well-posed boundary control system may be found in [8]. Among these possible choices, we select the impedance-passive input-output pairs of variables which result from the energy balance equation (see equation (II.7)) and dissipation inequality:

$$
\begin{aligned}
\frac{d \mathrm{H}_{d}}{d t} & =\int_{\mathcal{Z}}\left\langle\delta_{x} \mathrm{H}_{d}, \dot{x}\right\rangle=-\int_{\mathcal{Z}}\left\langle\delta_{x} \mathrm{H}_{d}, \mathcal{R}_{d} \delta_{x} \mathrm{H}_{d}\right\rangle+\int_{\partial \mathcal{Z}} \mathcal{B}_{d}\left(\delta_{x} \mathrm{H}_{d}, \delta_{x} \mathrm{H}_{d}\right) \\
& =-\int_{\mathcal{Z}}\left\langle\delta_{x} \mathrm{H}_{d}, \mathcal{R}_{d} \delta_{x} \mathrm{H}_{d}\right\rangle+\left\langle\tilde{u}_{2}, \tilde{y}_{2}\right\rangle \leq\left\langle\tilde{u}_{2}, \tilde{y}_{2}\right\rangle
\end{aligned}
$$

where $\langle\cdot, \cdot\rangle$ denotes the usual $L^{2}\left(\mathcal{Z}, \mathrm{R}^{n}\right)$ inner product on the whole spatial domain. The matching equation:

$$
g u_{1}=\left[\mathcal{J}_{d}(x, t)-\mathcal{R}_{d}(x, t)\right] \delta_{x} \mathrm{H}_{d}-[\mathcal{J}(x, t)-\mathcal{R}(x, t)] \delta_{x} \mathrm{H}
$$

then determines the "distributed" control action $g u_{1}$.

Remark IV.2: Contrarily to the "traditional" approach which consists in homogenizing the boundary control to embed it in the system state equation and then handling it as a distributed control, the method presented in this work reverses the idea. In other words, the distributed and boundary controls are used to transform the original system (II.1) into the target canonical PHS (IV.1) which still has a boundary control (usually not the same as the one in the original system).

Remark IV.3: A dissipative target PHS, that is with $\mathcal{R}_{d}>0$, is asymptotically stable even with an homogenous boundary condition $\tilde{u}_{2}=0$ (see equation IV.4). Otherwise, if $\mathcal{R}_{d} \geq 0$ is only positive semidefinite, a simple "boundary damping" injection of the form $\tilde{u}_{2}=-K_{p} \tilde{y}_{2}, K_{p}>0$ ensures the stabilization:

$$
\frac{d \mathrm{H}_{d}}{d t} \leq-K_{p}\left\langle\tilde{y}_{2}, \tilde{y}_{2}\right\rangle<0
$$


in the sense of Lyapunov stability.

\section{IV.2. Exponential stabilization with energy shaping and damping assignment control for a subclass of linear PHS}

We will now focus on a particular subclass of linear PHS, assuming that the energy function of the original system has the quadratic form $\mathrm{H}=\int_{\mathcal{Z}} \frac{1}{2} x^{T} \mathcal{Q} x$ (i.e. the system dynamics is linear) and that the canonical choice $\left(u_{2}, y_{2}\right)=\left.\left(\delta_{x} \mathrm{H}\right)\right|_{\partial \mathcal{Z}}$ of boundary input-output impedance-passive variables have been selected (cf. [18]). It may happen that the interconnection operator $\mathcal{J}$ has a suitable form which should remain unchanged in the target system. In fact, this is the most common case and the geometric interconnection structure of the actual model should not be changed in the target system unless some specific purpose is given since it affects the structural invariants and intrinsic dynamical behavior of the system. For instance, in the resistive diffusion equation example hereafter, the desired interconnection operator $\mathcal{J}_{d}$ is defined using the derivation operator:

$$
\mathcal{J}_{d}=\mathcal{J}=\left(\begin{array}{ll}
0 & 1 \\
1 & 0
\end{array}\right) \partial_{z}
$$

together with some given boundary conditions (see Section 3). In the case where the interconnection structure of the actual system can remain unchanged, the target system is obtained by using only "energy shaping" and/or "damping injection":

- $\mathrm{H}_{d}=\int_{\mathcal{Z}} \frac{1}{2} x^{T} \mathcal{Q}_{d} x=\int_{\mathcal{Z}} \frac{1}{2} x^{T}\left(\mathcal{Q}+\mathcal{Q}_{a}\right) x=\mathrm{H}+\mathrm{H}_{a} ; \mathcal{Q}_{d}>0$

- $\mathcal{R}_{d}=\mathcal{R}+\mathcal{R}_{a} \geq 0$ with $\mathcal{R}_{a}=\mathcal{R}_{a}^{T} \neq 0$

The new "passive" boundary input (and the corresponding conjugated output) of the target system is determined via the chosen Hamiltonian $\mathrm{H}_{d}$ and thus may be related with the original system boundary control $u_{2}$ :

$$
\begin{aligned}
\left(\begin{array}{c}
\tilde{u}_{2} \\
\tilde{y}_{2}
\end{array}\right)=\left(\begin{array}{c}
f_{\partial} \\
e_{\partial}
\end{array}\right) & =\left.\left(\delta_{x} \mathrm{H}\right)\right|_{\partial \mathcal{Z}} \\
& =\left.\left(\delta_{x} \mathrm{H}\right)\right|_{\partial \mathcal{Z}}+\left.\left(\delta_{x} \mathrm{H}_{a}\right)\right|_{\partial \mathcal{Z}} \\
& =\left(\begin{array}{c}
u_{2} \\
y_{2}
\end{array}\right)+\left.\left(\delta_{x} \mathrm{H}_{a}\right)\right|_{\partial \mathcal{Z}}
\end{aligned}
$$

This new boundary input $\tilde{u}_{2}$ is thus modified only by the energy shaping $\mathrm{H}_{a}$ and not influenced by the damping injection $\mathcal{R}_{a}$. Furthermore, in the considered linear case exponential stability will be achieved without any additional boundary control $\tilde{u}_{2}$. The stability of the target system may be proved via the first and second Arnold's stability theorems (see for instance [16]) by using a suitable norm. Here, the norm associated with the energy stored in the target system results in very simple calculations to prove the asymptotic (exponential) stability with respect to this norm. Indeed, assume that the energy function of the target system is the quadratic form $\mathrm{H}_{d}=\frac{1}{2} \int_{\mathcal{Z}} x^{T} \mathcal{Q}_{d} x=\|x\|_{\mathcal{Q}_{d}}^{2}$, then $\delta_{x} \mathrm{H}_{d}\left(x^{*}\right)=\mathrm{H}\left(x^{*}\right)=0$ at the equilibrium profile $x^{*}=0$. Considering no boundary control $\tilde{u}_{2}=0$ in the system 
(IV.1), the energy balance reads:

$$
\begin{aligned}
\frac{d}{d t} \mathrm{H}_{d} & =-\int_{\mathcal{Z}} x^{T} \mathcal{Q}_{d} \mathcal{R}_{d} \mathcal{Q}_{d} x \\
& =-\left\|\left(\mathcal{Q}_{d}\right)^{-1 / 2}\left(\mathcal{R}_{d}\right)^{1 / 2} \mathcal{Q}_{d} x\right\|_{\mathcal{Q}_{d}}^{2} \leq-\Gamma\|x\|_{\mathcal{Q}_{d}}^{2}
\end{aligned}
$$

where $\Gamma$ is the minimum singular value of $\left(\mathcal{Q}_{d}\right)^{-1 / 2}\left(\mathcal{R}_{d}\right)^{1 / 2} \mathcal{Q}_{d}$. In the case of a non-zero damping injection $\mathcal{R}_{d}>0$, we have $\Gamma>0$ and:

$$
\frac{d}{d t} \mathrm{H}_{d} \leq-\Gamma\|x\|_{\mathcal{Q}_{d}}^{2}=-\Gamma \mathrm{H}_{d}
$$

which proves exponential stability in the sense of Lyapunov with respect to the energy norm $\|\cdot\|_{\mathcal{Q}_{d}}$.

\section{Average IDA-PBC design with boundary backstepping stabilization}

The solution of the matching equation (IV.5) is far from trivial, among others due to the specific conditions that each "control parameter" $\left(\mathcal{J}_{d}, \mathcal{R}_{d}, \mathrm{H}_{d}\right)$ must satisfy. On the other hand, in many applications only a finite rank distributed control is available. This is the case for instance for the plasma control example investigated in this paper. Therefore, a "sensible" idea is to use these finite number of available degrees of freedom to match as much moments as possible in the matching equation. In the considered application case, the control rank is 1 and consequently we can only solve the matching equation in the average sense. Although the average solution of the matching equation may often be easily obtained, this approximation does not guarantee by itself the stability of the corresponding closed-loop system. Hence, we will make use of the boundary control to stabilize the matching error in the whole spatial domain. Although its influence inside the spatial domain is not instantaneous and direct as the one from the direct distributed control, it spreads along the whole spatial domain and can somehow correct the error coming from the distributed matching equation. In fact, thanks to the Volterra transformation (or backstepping boundary control, according to the terminology in [6]), the system stabilization will be recovered despite of the error in the matching equation. A relaxation in the matching equation solution is thus proposed, combined with an error stabilization using simultaneously the distributed and boundary control actions.

\section{V.1. Average solution for the matching equation with distributed control}

How to solve the matching equation (IV.5) and how to parametrize the solutions are major concerns in the IDA-PBC literature even for the control design of finite dimensional systems (cf. [14],[12]). Since in our case the distributed control is only finite rank, there is no solution in general for the matching equation in the infinite dimensional case. We present hereafter an approach to "solve" this problem in some approximate (average) sense. Indeed the scalar value of $u_{1}(t)$, not depending on the spatial coordinate $z$, may be isolated from the control spatial distribution 
$g(z, t)$ in the matching equation when computing moments for this distribution. For instance, the average value holds:

$$
\begin{aligned}
u_{1}(t) \int_{\mathcal{Z}} g(z, t) d z= & \int_{\mathcal{Z}}\left[\mathcal{J}_{d}(x, t)-\mathcal{R}_{d}(x, t)\right] \delta_{x} \mathrm{H}_{d} d z \\
& -\int_{\mathcal{Z}}[\mathcal{J}(x, t)-\mathcal{R}(x, t)] \delta_{x} \mathrm{H} d z
\end{aligned}
$$

The obtained control $u_{1}$ is the one which cancels the average value (on the whole spatial 1D domain $\mathcal{Z}$ ) of the residual for the matching equation. The idea could be extended to higher moments of the matching error residual in the case where several control variables are available.

Remark V.1: The previous solutions for the matching equation deduces the error from the average calculus. Therefore, one can't ensure to transform the original system into the target one, which is stable, with such derived control. The discussion then consists in knowing whether or not the boundary control $u_{2}$ can be used to stabilize this "matching error". The proposition in the next section will exploit this idea.

\section{V.2. Exponential stability for the error model using boundary control}

We describe in the next proposition an extended version of IDA-PBC for infinite dimensional PHS, using both finite rank distributed control and boundary backstepping control actions.

Proposition V.2: We consider the closed-loop system obtained with the average finite rank distributed control:

$$
\dot{x}=[\mathcal{J}-\mathcal{R}] \mathcal{Q} x+g u_{1}=\left[\mathcal{J}_{d}-\mathcal{R}_{d}\right] \mathcal{Q}_{d} x+\epsilon(z)
$$

where $\epsilon(x, z)$ is the matching error in (IV.5). We define the Volterra state space transformation:

$$
w=x-\int_{0}^{z} k(z, y) x(y, t) d y
$$

where the functional matrix $k(z, y) \in \mathcal{M}_{n}(\mathrm{R})$ is the Volterra kernel with $z \in \mathcal{Z}$ and $y \in[0, z]$.

The original system turns into a new system which is stable, if there exists a corresponding kernel $k(z, y)$ satisfying:

$$
\begin{aligned}
0 & =\epsilon(z)-\int_{0}^{z} k(z, y)\left[\mathcal{J}_{d}-\mathcal{R}_{d}\right] \mathcal{Q}_{d}(y) x(y) d y \\
& -\int_{0}^{z} k(z, y) \epsilon(y) d y+\left[\mathcal{J}_{d}-\mathcal{R}_{d}\right] \mathcal{Q}_{d} \int_{0}^{z} k(z, y) x(y, t) d y
\end{aligned}
$$

with the boundary condition $\epsilon(0)=0$. The boundary control $u_{2}$ in (II.1) becomes then:

$$
u_{2}=\left.\left(\mathcal{Q}\left(w+\int_{0}^{z} k(z, y) x(y, t) d y\right)\right)\right|_{z=1}
$$


Proof V.3: Combining these previous equations (V.2-V.4), one gets:

$$
\begin{aligned}
\dot{w} & =\dot{x}-\int_{0}^{z} k(z, y) \dot{x}(y, t) d y \\
& =\left[\mathcal{J}_{d}-\mathcal{R}_{d}\right] \mathcal{Q}_{d} w
\end{aligned}
$$

The system (V.6), without boundary control ( $\left.u_{2}^{\prime}=\left.[1,0] \mathcal{Q}_{d} w\right|_{z=1} \equiv 0\right)$, is asymptotically stable with $\mathcal{R}_{d}>0$, according to the balance equation in (IV.8IV.9).

Remark V.4: The system (V.6) is equivalent to the original system with the boundary control $u_{2}$ defined in (V.5). The Volterra transformation kernel $k(z, y)$ describes the way the boundary control $u_{2}$ propagates inside the system domain $\mathcal{Z}$, and how it allows to compensate the matching error $\epsilon(x)$ and to stabilize the closed-loop system when the condition in (V.4) is satisfied.

Furthermore, it is easy to add a boundary damping via $u_{2}^{\prime}$ defined as $u_{2}^{\prime}=-K_{p} y_{2}^{\prime}$ in order to improve the stabilization of the system (V.6), as well as the one of our original system (II.1).

Remark V.5: The case where $\epsilon(z)=0$ falls into the classical IDA-PBC design. Moreover, equation (V.4) leads to a specific choice of controller parameters $\left(\mathcal{J}_{d}, \mathcal{R}_{d}, \mathrm{H}_{d}\right)$ such that:

$$
-\int_{0}^{z} k(z, y)\left[\mathcal{J}_{d}-\mathcal{R}_{d}\right] \mathcal{Q}_{d}(y) x(y) d y+\left[\mathcal{J}_{d}-\mathcal{R}_{d}\right] \mathcal{Q}_{d}(z) \int_{0}^{z} k(z, y) x(y, t) d y=0
$$

The presented methodology may be summarized in the following way:

$$
\begin{aligned}
& \text { Original system } \begin{cases}\dot{x} & =[\mathcal{J}-\mathcal{R}] \mathcal{Q} x+g u_{1} \\
\left(\begin{array}{l}
u_{2} \\
y_{2}
\end{array}\right) & =\left.\mathcal{Q} x\right|_{z=1} \\
\mathrm{H}(x) & =\int_{\mathcal{Z}} \frac{1}{2} x^{T} \mathcal{Q} x\end{cases} \\
& \stackrel{u_{1} \mathrm{IDA}}{\longrightarrow} \begin{cases}\dot{x} & =\left[\mathcal{J}_{d}-\mathcal{R}_{d}\right] \mathcal{Q}_{d} x+\epsilon(z) \\
\left(\begin{array}{c}
\tilde{u}_{2} \\
\tilde{y}_{2}
\end{array}\right) & =\left.\mathcal{Q}_{d} x\right|_{z=1} \\
\mathrm{H}_{d}(x) & =\int_{\mathcal{Z}} \frac{1}{2} x^{T} \mathcal{Q}_{d} x\end{cases} \\
& w=x-\int_{0}^{z} k(z, y) x(y, t) \mathrm{d} y \begin{cases}\dot{w} & =\left[\mathcal{J}_{d}-\mathcal{R}_{d}\right] \mathcal{Q}_{d} w \\
\left(\begin{array}{c}
u_{2}^{\prime} \\
y_{2}^{\prime}
\end{array}\right) & =\left.\mathcal{Q}_{d} w\right|_{z=1} \\
\mathrm{H}_{d w}(w) & =\int_{\mathcal{Z}} \frac{1}{2} w^{T} \mathcal{Q}_{d} w\end{cases}
\end{aligned}
$$

We will now investigate the new matching equations to determine the system controls $u_{1}$ and $u_{2}$ together with the appropriate choice of the kernel $k(z, y)$ for the Volterra transformation and the IDA-PBC parameters $\left(\mathcal{J}_{d}, \mathcal{R}_{d}, \mathrm{H}_{d}\right)$. 


\section{V.3. Solution proposal for the kernel equation}

In this subsection, we will look for a particular solution $k \in \mathcal{M}_{2}(\mathrm{R})$ of the kernel equation which applies only for the considered example presented in Section III. For the sake of notational compactness, we will make use of the notation

$$
\mathcal{J}_{d}=\mathcal{J}=\left(\begin{array}{ll}
0 & 1 \\
1 & 0
\end{array}\right) \partial_{z}=i \partial_{z}
$$

The last term in the kernel equation (V.4) may be transformed:

$$
\begin{aligned}
{\left[\mathcal{J}_{d}-\mathcal{R}_{d}\right] \mathcal{Q}_{d} \int_{0}^{z} k(z, y) x(y, t) \mathrm{d} y=} & i \mathcal{Q}_{d}(z) k(z, z) x(z)+\int_{0}^{z} i \partial_{z}\left(\mathcal{Q}_{d}(z) k(z, y)\right) x(y) \mathrm{d} y \\
& -\int_{0}^{z} \mathcal{R}_{d}(z) \mathcal{Q}_{d}(z) k(z, y) x(y) \mathrm{d} y
\end{aligned}
$$

since $\partial_{z} \int_{0}^{z} f(z, y) \mathrm{d} y=f(z, z)+\int_{0}^{z} \partial_{z} f(z, y) \mathrm{d} y$. The second term in the of (V.4) becomes:

$$
\begin{aligned}
-\int_{0}^{z} k(z, y)\left[\mathcal{J}_{d}-\mathcal{R}_{d}\right] \mathcal{Q}_{d}(y) x(y) \mathrm{d} y= & -k(z, z) i \mathcal{Q}_{d}(z) x(z)+k(z, 0) i \mathcal{Q}_{d}(0) x(0) \\
& +\int_{0}^{z} \partial_{y} k(z, y) i \mathcal{Q}_{d}(y) x(y) \mathrm{d} y \\
& +\int_{0}^{z} k(z, y) \mathcal{R}_{d}(y) \mathcal{Q}_{d}(y) x(y) \mathrm{d} y
\end{aligned}
$$

The remaining terms in (V.4) may be written:

$$
\epsilon(z)-\int_{0}^{z} k(z, y) \epsilon(y) d y=\epsilon(0)+\int_{0}^{z}\left(\partial_{y} \epsilon(y)-k(z, y) \epsilon(y)\right) d y
$$

using the fact that for any absolutely continuous function $f(z)$ in $\mathcal{Z}$,

$$
f(z)=f(0)+\int_{0}^{z} \partial_{y} f(y) \mathrm{d} y
$$

Therefore equation (V.4) is satisfied when the three following conditions hold:

$$
\begin{cases}i \mathcal{Q}_{d}(z) k(z, z) x(z)-k(z, z) i \mathcal{Q}_{d}(z) x(z) & =0(a) \\ i \partial_{z} \mathcal{Q}_{d}(z) k(z, y) x(y)+i \mathcal{Q}_{d}(z) \partial_{z} k(z, y) x(y)-\mathcal{R}_{d}(z) \mathcal{Q}_{d}(z) k(z, y) x(y) & =0(b) \\ \partial_{y} \epsilon(y)-k(z, y) \epsilon(y)+\partial_{y} k(z, y) i \mathcal{Q}_{d}(y) x(y)+k(z, y) \mathcal{R}_{d}(y) \mathcal{Q}_{d}(y) x(y) & =0(c)\end{cases}
$$

Note also that the boundary condition:

$$
\epsilon(0)+k(z, 0) i \mathcal{Q}_{d}(0) x(0)=0
$$

is satisfied since the following boundary condition holds since $\left.\epsilon\right|_{z=0}=\left.x\right|_{z=0}=0$. Hence, in order to transform the original system (V.8) into the target system (V.10), it is sufficient to prove the existence of a functional matrix $k(z, y)$ such that equation set (V.14) is satisfied. The idea is then to choose specific design parameters $\left(\mathcal{Q}_{d}, \mathcal{R}_{d}\right)$ for which this system of equations is easy to solve. In the 
sequel the parameters $\left(\mathcal{Q}_{d}, \mathcal{R}_{d}\right)$ are therefore computed together with the kernel $k$.

\section{V.3.1. Existence of a kernel $k(z, y)=k(y)$}

We look for a particular solution $k(z, y)=k(y)$ of equation (V.14). For such a solution, equation $(b)$ states that the design parameters $\left(\mathcal{Q}_{d}, \mathcal{R}_{d}\right)$ must satisfy:

$$
i \partial_{z} \mathcal{Q}_{d}(z)-\mathcal{R}_{d}(z) \mathcal{Q}_{d}(z)=0
$$

This condition will be investigated later in Subsection V.3.2. Equations $(a)$ and $(c)$ in (V.14) suggest to choose:

$$
k(y)=\left(\begin{array}{cc}
k_{1} & k_{12} \\
k_{21} & k_{2}
\end{array}\right) \text { and } \mathcal{Q}_{d}=\mathcal{Q}_{d}^{T}=\left(\begin{array}{cc}
q_{1} & q_{12} \\
q_{12} & q_{2}
\end{array}\right)>0
$$

Indeed equation $(a)$ then becomes:

$$
\begin{array}{r}
i \mathcal{Q}_{d}(z) k(z, z)-k(z, z) i \mathcal{Q}_{d}(z)=0 \\
\left(\begin{array}{ll}
0 & 1 \\
1 & 0
\end{array}\right)\left(\begin{array}{cc}
q_{1} & q_{12} \\
q_{12} & q_{2}
\end{array}\right)\left(\begin{array}{cc}
k_{1} & k_{12} \\
k_{21} & k_{2}
\end{array}\right)-\left(\begin{array}{cc}
k_{1} & k_{12} \\
k_{21} & k_{2}
\end{array}\right)\left(\begin{array}{ll}
0 & 1 \\
1 & 0
\end{array}\right)\left(\begin{array}{cc}
q_{1} & q_{12} \\
q_{12} & q_{2}
\end{array}\right)=0 \\
\Leftrightarrow \begin{cases}k_{1}=k_{2} \\
q_{2} k_{21}=k_{12} q_{1}\end{cases}
\end{array}
$$

and equation (c) in (V.14) transforms into:

$$
\partial_{y} k(y) \mathcal{A}(y)+k(y) \mathcal{D}(y)+\mathcal{C}(y)=0
$$

where

$$
\begin{cases}\mathcal{A}(y)=\left[\mathcal{A}_{1}(y), \mathcal{A}_{2}(y)\right]^{T} & =i \mathcal{Q}_{d}(y) x(y) \\ \mathcal{D}(y)=\left[\mathcal{D}_{1}(y), \mathcal{D}_{2}(y)\right]^{T} & =-\epsilon(y)+\mathcal{R}_{d}(y) \mathcal{Q}_{d}(y) x(y) \\ \mathcal{C}(y)=\left[\mathcal{C}_{1}(y), \mathcal{C}_{2}(y)\right]^{T} & =\partial_{y} \epsilon(y)\end{cases}
$$

We can develop equation (V.18) with the condition in (V.17) such that:

$$
\begin{cases}\partial_{y} k_{1} \mathcal{A}_{1}+k_{1} \mathcal{D}_{1}+\partial_{y} k_{12} \mathcal{A}_{2}+k_{12} \mathcal{D}_{2}+\mathcal{C}_{1} & =0(d) \\ \partial_{y} k_{1} \mathcal{A}_{2}+k_{1} \mathcal{D}_{2}+\partial_{y} k_{12}\left(q_{2}^{-1} q_{1}\right) \mathcal{A}_{1}+k_{12}\left(\partial_{y}\left(q_{2}^{-1} q_{1}\right) \mathcal{A}_{1}+\left(q_{2}^{-1} q_{1}\right) \mathcal{D}_{1}\right)+\mathcal{C}_{2} & =0(e)\end{cases}
$$

The linear combinations of $(d)$ and $(e)$ in the previous equation, such as $\left(\mathcal{A}_{2}(d)-\mathcal{A}_{1}(e)\right)$ and $\left(\mathcal{D}_{2}(d)-\mathcal{D}_{1}(e)\right)$ lead to two distinguished differential equations for $k_{1}$ and $k_{12}$ in the form of:

$$
\partial_{y} k_{i}+\gamma_{i} k_{i}+\lambda_{i}=0, \quad i=1 \text { or } 12
$$

where $\gamma_{i}$ and $\lambda_{i}$ are computed from $\mathcal{A}(y), \mathcal{D}(y)$ and $\mathcal{C}(y)$. Note that $\operatorname{rank}([\mathcal{A}(y), \mathcal{D}(y), \mathcal{C}(y)])=2$, thus there exists an explicit solution $k$ for (V.18) with the form:

$$
\left\{\begin{array}{l} 
\begin{cases}k_{1} & =k_{2} \\
q_{2} k_{21} & =k_{12} q_{1}\end{cases} \\
k_{i}(y)=e^{-\phi_{i}(y)}\left(\int_{0}^{y}-\lambda_{i}(\xi) e^{\phi_{i}(\xi)} d \xi+\kappa_{i}\right) \quad i=1 \text { or } 12
\end{array}\right.
$$


with $\phi_{i}(y)=\int_{0}^{y} \gamma_{i}(\xi) \mathrm{d} \xi$, and where $\kappa_{i}$ is an integration constant depending on the initial condition.

Remark V.6: To find the general solution $k(z, y)$ for the kernel matching equation (V.14) is a real challenge. However finding a particular kernel $k(y)$ for the corresponding backstepping transformation $w=x-\int_{0}^{z} k(y) x(y) \mathrm{d} y$ is in fact sufficient to solve the matching problem.

\section{V.3.2. The corresponding choices for the design parameters $\left(\mathcal{R}_{d}, \mathcal{Q}_{d}\right)$}

Again we consider the closed loop energy and dissipation matrices

$$
\mathcal{R}_{d}=\left(\begin{array}{cc}
r_{1} & r_{12} \\
r_{12} & r_{2}
\end{array}\right)>0 \text { and } \mathcal{Q}_{d}=\left(\begin{array}{cc}
q_{1} & q_{12} \\
q_{12} & q_{2}
\end{array}\right)>0
$$

Note that the case $q_{12}=0$ is not compatible with the chosen closed loop interconnection structure $\mathcal{J}_{d}=i \partial_{z}$. We will therefore consider the simplest case with $r_{1}, r_{2}>0 ; r_{12}=0$ and $q_{1} q_{2}-q_{12}^{2}>0$. Then the compatibility equation (V.16) for $\left(\mathcal{R}_{d}, \mathcal{Q}_{d}\right)$ holds if

$$
\begin{cases}\partial_{z} q_{12} & =r_{1} q_{1}=r_{2} q_{2} \\ \partial_{z} q_{1} & =r_{2} q_{12} \\ \partial_{z} q_{2} & =r_{1} q_{12}\end{cases}
$$

For fixed chosen values of $r_{1}, r_{2}$, these conditions result in:

$$
\frac{\partial_{z} q_{1}}{\partial_{z} q_{2}}=\frac{q_{1}}{q_{2}}=\frac{r_{2}}{r_{1}}=\alpha(z)>0
$$

Assume now that $q_{2}=\beta(z)$ and $\partial_{z} \beta(z) \neq 0$. From (V.23), we get:

$$
\left\{\begin{array}{ll}
q_{1}(z) & =\alpha(z) \beta(z) \\
\partial_{z} q_{1}(z) & =\alpha(z) \partial_{z} \beta(z)
\end{array} \Rightarrow \alpha(z)=\alpha\right. \text { constant }
$$

with the additional condition $-\sqrt{\alpha} r_{1}<0<\frac{\partial_{z} q_{1}}{q_{1}}<\sqrt{\alpha} r_{1}$ which guarantees $\mathcal{Q}_{d}>0$. Hence we get

$$
q_{1}(z)=C e^{\int_{0}^{z}\left(\sqrt{\alpha} r_{1}-\epsilon\right) d z}
$$

where $C$ and $\epsilon=\sqrt{\alpha} r_{1}-\frac{\partial_{z} q_{1}}{q_{1}}>0$ are tuning constants.

\section{V.3.3. Volterra inverse transformation}

Stability of the closed loop system with respect to the original coordinates $x$ requires that the inverse backstepping transformation exists and is bounded. To prove this is the purpose of the current subsection. Assuming that the Volterra inverse transformation takes the form [6]:

$$
x(z)=w(z)+\int_{0}^{z} l(z, y) w(y) d y
$$


from the matching equations (V.8-V.10), we may derive a set of conditions for $l(z, y)$ which are similar to the ones for $k(z, y)$ :

$$
\begin{cases}i \mathcal{Q}_{d}(z) l(z, z) w(z)-l(z, z) i \mathcal{Q}_{d}(z) w(z) & =0(a) \\ i \partial_{z} \mathcal{Q}_{d}(z) l(z, y) w(y)+i \mathcal{Q}_{d}(z) \partial_{z} l(z, y) w(y)-\mathcal{R}_{d}(z) \mathcal{Q}_{d}(z) l(z, y) w(y) & =0(b) \\ \partial_{y} \epsilon(y)+\partial_{y} l(z, y) i \mathcal{Q}_{d}(y) w(y)+l(z, y) \mathcal{R}_{d}(y) \mathcal{Q}_{d}(y) w(y) & =0(c)\end{cases}
$$

Using the particular choice of control parameters $\mathcal{Q}_{d}$ and $\mathcal{R}_{d}$ defined in subsection V.3.2, and defining

$$
l(z, y)=l(y)=\left(\begin{array}{cc}
l_{1} & l_{12} \\
l_{21} & l_{2}
\end{array}\right)
$$

we get:

$$
\begin{cases}i \mathcal{Q}_{d}(z) l(z)-l(z) i \mathcal{Q}_{d}(z) & =0 \\ \partial_{y} l(y) \mathcal{A}(y)+l(y) \mathcal{D}_{l}(y)+\mathcal{C}(y) & =0\end{cases}
$$

with $\mathcal{D}_{l}(y)=\mathcal{R}_{d}(y) \mathcal{Q}_{d}(y) w(y)$. The solution for $l(y)$ is similar to the one for $k(y)$ :

$$
\left\{\begin{array}{l} 
\begin{cases}l_{1} & =l_{2} \\
q_{2} l_{21} & =l_{12} q_{1}\end{cases} \\
l_{i}(y)=e^{-\phi_{l i}(y)}\left(\int_{0}^{y} \lambda_{i}(\xi) e^{\phi_{l i}(\xi)} d \xi+\kappa_{l i}\right) \quad i=1 \text { or } 12
\end{array}\right.
$$

where $\kappa_{l i}$ is a constant of integration depending on the initial condition.

Now we have to prove that this inverse Volterra transformation is bounded. For this we will make use of the usual norm in $L^{2}\left(\mathcal{Z}, \mathrm{R}^{n}\right)$. From (V.26), we have:

$$
\begin{aligned}
\|x\|^{2} & =\int_{\mathcal{Z}}\left|w+\int_{0}^{z} l(y) w(y) d y\right|^{2} d \xi \\
& \leq \int_{\mathcal{Z}}|w|^{2} d \xi+\int_{\mathcal{Z}}\left(\int_{0}^{z}|l(y)|^{2}|w(y)|^{2} d y\right) d \xi \\
& \leq\|w\|^{2}+\Gamma^{2}\left(\int_{0}^{z} \int_{\mathcal{Z}}|w(y)|^{2} d \xi d y\right) \\
& \leq\|w\|^{2}+\Gamma^{2} \int_{0}^{z}\|w(y)\|^{2} d y
\end{aligned}
$$

where $\Gamma$ denotes the maximum singular value of $l(y)$ in (V.29) which is bounded when $\mathcal{R}_{d}$ and $\mathcal{Q}_{d}$ are chosen from Subsection V.3.2. 


\section{Example and simulation results}

The simulation is demonstrated for the resistive diffusion tokamak plasma studied in Section III. The RAPTOR code (cf. [4]), the tokamak simulator with the TCV configuration is used. The system states are the toroidal electric and poloidal magnetic flows $x=\left(\bar{D}_{\phi}, \bar{B}_{\theta}\right)^{T}$ for $n=2$. The control signals are two scalars: the heating power $u_{1}=\delta P_{\text {ext }}(t)$ of the non-inductive current $\bar{J}_{\text {ext }}$ and the loop voltage $u_{2}=\delta V_{\text {loop }}(t)$. Note that $P_{\text {ext }}(t)$ is the power difference between the co-current $P_{A}(t)$ and counter-current $P_{B}(t)$ sources.

In this work, we consider that the whole state is measured or computable from measurements. Two reference values for safety factor $q$ are defined at the radial relative coordinates $z=0.1$, and $z=0.4$. The feedforward calculus is firstly computed to give the feedforward control and the equilibrium $q$-profile, corresponding to the references and taking into account actuators limits and non-linearities. The boundary IDA-PBC control presented in the previous section defines the feedback signals with the choice of desired dissipation:

$$
\mathcal{R}_{d}=\left(\begin{array}{cc}
r_{1} & 0 \\
0 & r_{2}
\end{array}\right)>0
$$

where $r_{1}=\frac{C_{3}}{\eta}$ and the ratio $\alpha>0$ is fixed. According to Subsection V.3.2, $\mathcal{Q}_{d}$ is derived from the tuned dissipation $\mathcal{R}_{d}$ and the kernel $k(y)$ is then computed from equation (V.21). We explicit hereafter the boundary control $u_{2}$ derived from these tuning parameters. From the original system in (V.8), the boundary variables are:

$$
\left(\begin{array}{l}
u_{2} \\
y_{2}
\end{array}\right)=\left.\mathcal{Q} x\right|_{z=1}
$$

with the boundary control $u_{2}=\delta V_{\text {loop }}(t)$ and the measured plasma current $y_{2}=$ $\delta I_{p}$. We have:

$$
\begin{aligned}
u_{2} & =\left.[1,0] \mathcal{Q} x\right|_{z=1} \\
& =[1,0] \mathcal{Q}\left(\left.w\right|_{z=1}+\int_{0}^{1} k(y) x(y) \mathrm{d} y\right)
\end{aligned}
$$

In the case of homogenous boundary condition in the target system $\left(u_{2}^{\prime}=\right.$ $\left.\left.[1,0] \mathcal{Q}_{d} w\right|_{z=1} \equiv 0\right)$, the boundary control is simply (cf. [6]):

$$
u_{2}=[1,0] \mathcal{Q} \int_{0}^{1} k(y) x(y) \mathrm{d} y
$$

Hereafter, a boundary damping $u_{2}^{\prime}=-K_{p} y_{2}^{\prime}, K_{p}>0$ is often considered to improve 
the stability of the closed-loop system:

$$
\begin{aligned}
\left(\begin{array}{l}
u_{2} \\
y_{2}
\end{array}\right) & =\mathcal{Q}\left(\left.w\right|_{z=1}+\int_{0}^{1} k(y) x(y) \mathrm{d} y\right) \\
& =\mathcal{Q}\left(\mathcal{Q}_{d}^{-1}\left(\begin{array}{c}
u_{2}^{\prime} \\
y_{2}^{\prime}
\end{array}\right)+\int_{0}^{1} k(y) x(y) \mathrm{d} y\right) \\
& =\mathcal{Q}\left(\mathcal{Q}_{d}^{-1}\left(\begin{array}{c}
-K_{p} \\
1
\end{array}\right) y_{2}^{\prime}+\int_{0}^{1} k(y) x(y) \mathrm{d} y\right)
\end{aligned}
$$

since $\left(\begin{array}{l}u_{2}^{\prime} \\ y_{2}^{\prime}\end{array}\right)=\left.\mathcal{Q}_{d} w\right|_{z=1}$ from equation (V.10). The $y_{2}^{\prime}$ value is derived from the measured value of $y_{2}$ :

$$
y_{2}=[0,1] \mathcal{Q}\left(\mathcal{Q}_{d}^{-1}\left(\begin{array}{c}
-K_{p} \\
1
\end{array}\right) y_{2}^{\prime}+\int_{0}^{1} k(y) x(y) \mathrm{d} y\right)
$$

and finally, the boundary control with boundary damping is:

$$
u_{2}=[1,0] \mathcal{Q}\left(\mathcal{Q}_{d}^{-1}\left(\begin{array}{c}
-K_{p} \\
1
\end{array}\right) y_{2}^{\prime}+\int_{0}^{1} k(y) x(y) \mathrm{d} y\right)
$$

The Figures 3 shows the results obtained with the proposed control laws. The controller starts at $t=0.45 \mathrm{~s}$ with the initial values $\left(P_{\text {ext }}, V_{\text {loop }}\right)_{\text {init }}=(0,-0.65 \mathrm{~V})$, whereas at $z=(0.1,0.4)$ the reference of $q$ profile is set as $\left(q_{0.1}, q_{0.4}\right)=(0.85,1.25)$. Then at $t=1.2 s$, the reference is changed to $\left(q_{0.1}, q_{0.4}\right)=(0.95,1.25)$. The control signals and $q$-profile correspond to the boundary IDA-PBC case with both $\left(u_{1}, u_{2}\right)$.

The system errors at $z=0.1$ and $z=0.4$ are examined. The feedforward brings the $q$-profile near to the reference values, but there are still significant static errors coming from the linearization and the errors on the system parameter measurements. Using only $u_{1}$ obviously leads to a better result in comparing with the feedforward control but it can't optimize the system errors. The boundary IDAPBC control $\left(u_{1}, u_{2}\right)^{T}$ gives better results at both considered positions. The whole $q$-profile at $t=1.5 \mathrm{~s}$ also shows very small difference between the reference and the system state.

Furthermore, one can also use an integrator (defined such as in [14]) to eliminate the static errors (see the result in Figure 4). However, it must be noticed that this may be quite expensive (large actuator energy cost) only to get rid of a small static error and that, in practice, a small gap on $q$ - profile is still acceptable since it doesn't change much the physical properties of the system.

Remark VI.1: The robustness analysis is studied in [22]. Generally speaking, in this studied case, the robustness of the controller must be defined with respect two kinds of uncertainties: the major uncertainties (and model errors) on the system dissipation $\mathcal{R}$ (resulting from poor estimations for the plasma resistivity $\eta(z, t)$ ), uncertainties related to the linearization assumption made in the derivation the feedforward control and in the subsequent estimation of the bootstrap current $\bar{J}_{b s}(z, t)$ (the bootstrap current is taken account in the feedforward generation). According to [2], the system will remain stable as long as the desired dissipation is still positive and high enough to compensate the bounded perturbations. Moreover, a "strong" enough boundary damping effect can help to overcome these 

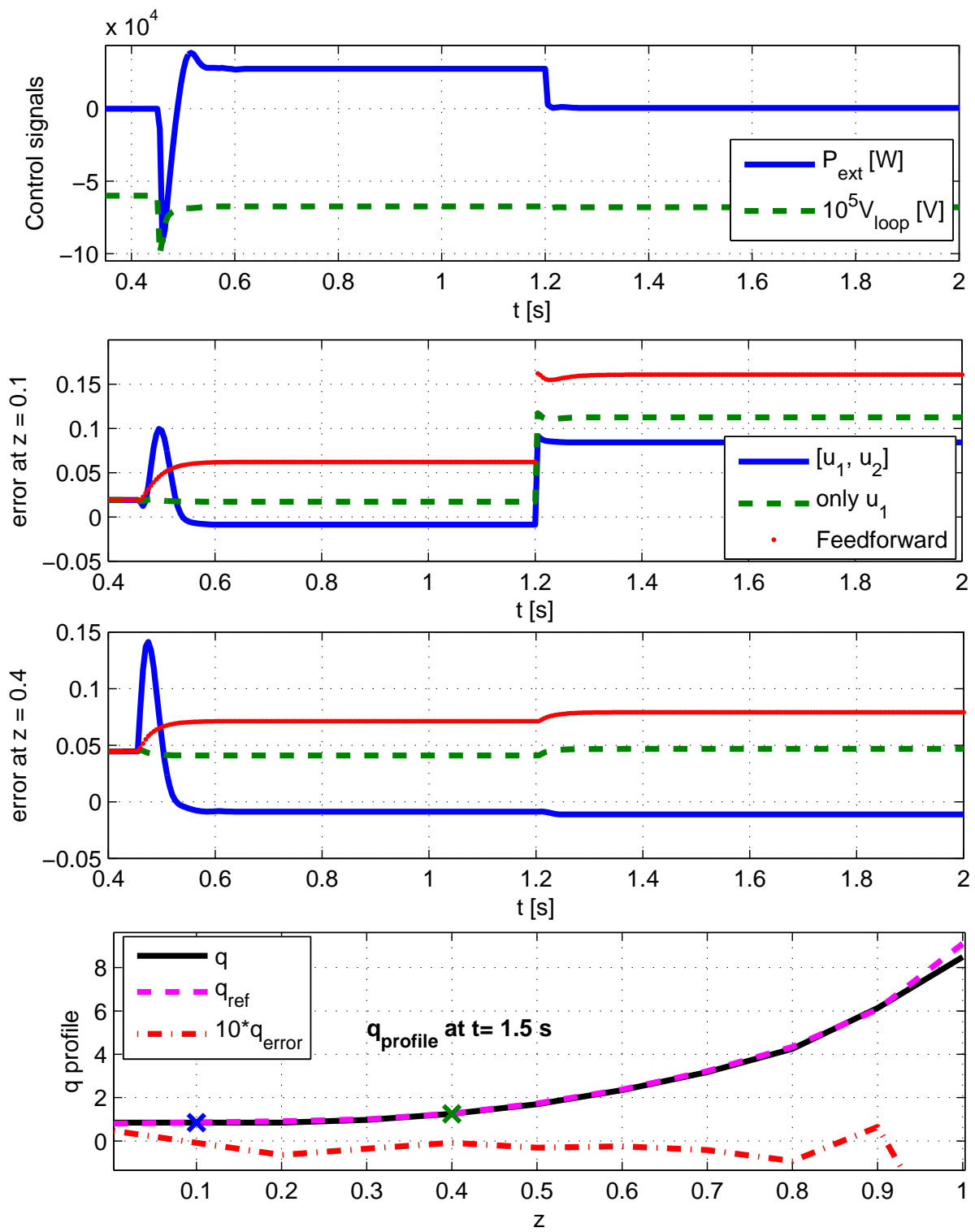

Figure 3. Simulation result of boundary IDA-PBC control for infinite dimensional resistive diffusion equation in Tokamaks

disturbances.

\section{Conclusion}

In this paper, a geometric reduction method for 3D systems of balance equations with spatial symmetries has been proposed. It has been applied to the set of electromagnetic balance equations which describes the plasma dynamics in a tokamak. A corresponding 1D port Hamiltonian model has been obtained - equivalent to the frequently used resistive diffusion equation control model - which inherits the suitable geometric structure for passivity based control from the 3D model. Then a boundary IDA-PBC control design for infinite dimensional PHS has been investigated. A distributed control has been determined using the "usual" IDA-PBC matching principle, but only in an average sense. Then a boundary control has 

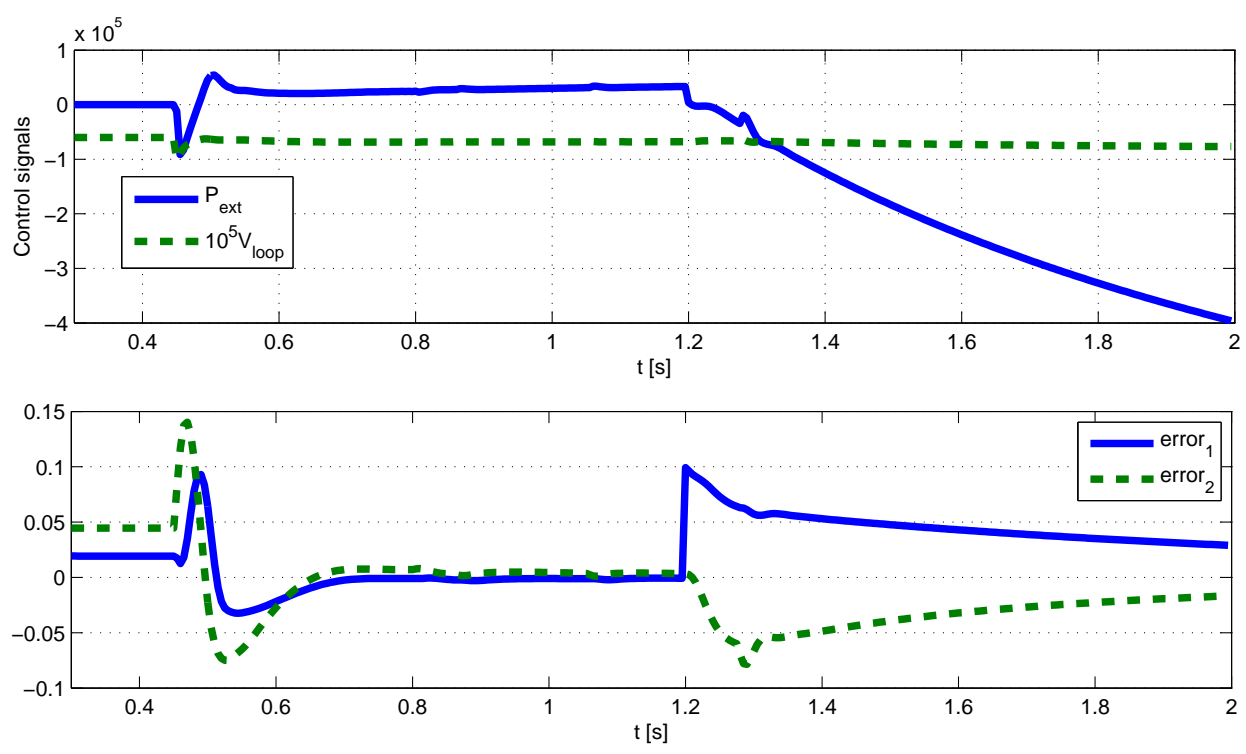

Figure 4. Simulation result of boundary IDA-PBC control with integrator

been to compensate the matching error and stabilizes the error system exponentially. A Volterra transformation (sometimes called backstepping) has been used to prove the stability of the target system.

The design approach has been applied to the previously obtained 1D resistive diffusion problem, using both a distributed finite rank control $\left(u_{1}\right)$ and a boundary control $\left(u_{2}\right)$. The matching of the resulting controlled system with the asymptotically stable target system has been proved. This is not the case when we use some average matching method for the infinite dimensional system or any finite dimensional IDA-PBC controller.

The proposed controller has been tested on the RAPTOR simulation tool developed for the TCV experiment at CRPP (EPFL). Numerical experiments show that indeed asymptotic convergence is reached with this feedback control. Moreover the controller is shown to be robust against the errors on the resistivity and on the non inductive current radial shape which are the two major physical uncertainties in this system.

\section{References}

[1] F.B. Argomedo, C. Prieur, E. Witrant, and S. Brémond. A strict control lyapunov function for a diffusion equation with time-varying distributed coefficients. IEEE Transactions on Automatic Control (2012), 2012.

[2] M. Becherif and E. Mendes. Stability and robustness of disturbed-port controlled hamiltonian systems with dissipation. In 16th IFAC world congress, Prague, Czech Republic, July 2005.

[3] J. Blum. Numerical Simulation and Optimal Control in Plasma Physics. GauthierVillars, New York, 1989.

[4] F. Felici, O. Sauter, S. Coda, B.P. Duval, T.P. Goodman, J.M. Moret, J.I. Paley, and the TCV Team. Real-time physics-model-based simulation of the current density profile in tokamak plasmas. Nuclear Fusion, 51, 2011.

[5] O. Gaye, E. Moulay, S. Brémond, L. Autrique, R. Nouailletas, and Y. Orlov. Sliding mode stabilization of the current profile in tokamak plasmas. Conference on Decision and Control (2011), Orlando, Florida, USA, December 2011. 
[6] M. Krstic and A. Smyshlyaev. Boundary control of PDEs: A course on Backstepping designs. Advances in Design and Control, SIAM, Philadelphia, 2008.

[7] A. Macchelli. Boundary energy shaping of linear distributed port-hamiltonian systems. European Journal of Control, 19:521-528, 2013.

[8] A. Macchelli, A.J. van der Schaft, and C. Melchiorri. Port hamiltonian formulation of infinite dimensional systems. i. modeling. Proc. 50th IEEE Conference on Decisions and Control (CDC04), 2004.

[9] A. Macchelli, A.J. van der Schaft, and C. Melchiorri. Port hamiltonian formulation of infinite dimensional systems. ii. boundary control by interconnection. 43rd IEEE Conference on Decisions and Control (CDC04), 2004.

[10] B. Maschke and A.J. van der Schaft. Compositional modelling of distributed-parameter systems, Advanced Topics in Control Systems Theory. Lecture Notes from FAP 2004. Lecture Notes on Control and Information Sciences. Springer-Verlag, London, 2005 (115-154).

[11] R. Ortega, A.J. van der Schaft, F. Castanos, and A. Astolfi. Control by interconnection and standard passivity-based control of port-hamiltonian systems. IEEE Trans. on Automatic Control, 53(11):2527-2542, 2008.

[12] R. Ortega, A.J. van der Schaft, B. Maschke, and G. Escobar. Interconnection and damping assignment: passivity-based control of port-controlled Hamiltonian systems. Automatica, 38(4):585-596, 2002.

[13] H. Ouarit, S. Brémond, R.Nouailletas, E.Witrant, and L.Autrique. Model based predictive control of tokamak plasma current profile. 26th Symposium on Fusion Technology (SOFT 2010), Porto, Portugal, Sept Sep 2010.

[14] R.Ortega and E.Garcia-Canseco. Interconnection and damping assignement passivitbased control: A survey. European Journal of Control, 110, 2003.

[15] M. Schöberl and A. Siuka. On casimir functionals for infinite-dimensional porthamiltonian control systems. IEEE Transactions on Automatic Control, 7(58):18231828, 2013.

[16] G.E. Swaters. Introduction to Hamiltonian fluid dynamics and stability theory. Chapman \& Hal/CRC, 2000.

[17] A.J. van der Schaft and B.M. Maschke. Hamiltonian formulation of distributed parameter systems with boundary energy flow. Journal of Geometry and Physics, 42:166174,2002

[18] J. Villegas, H. Zwart, Y. Le Gorrec, and B.M. Maschke. Stability and stabilization of a class of boundary control systems. IEEE Transaction On Automatic Control, 54:142-147, January 2009.

[19] Ngoc Minh Trang Vu, Laurent Lefèvre, and Bernhard Maschke. A structured control model for the thermo-magneto-hydrodynamics of plasmas in tokamaks. Mathematical and Computer Modelling of Dynamical Systems, (DOI: 10.1080/13873954.2016.1154874):1-26, 2016.

[20] Trang.N.M. Vu, L. Lefèvre, and B. Maschke. Port-hamiltonian formulation for systems of conservation laws: application to plasma dynamics in tokamak reactors. 4th IFAC Workshop on Lagrangian and Hamiltonian Methods for Non Linear Control, Bertinoro, Italy, August 29-31 2012.

[21] Trang.N.M. Vu, L. Lefèvre, R. Nouailletas, and S. Brémond. An ida-pbc approach for the control of $1 \mathrm{~d}$ plasma profile in tokamaks. 52nd IEEE Conference on Decision and Control (2013), Florence, Italy, December 10-13 2013.

[22] Trang.N.M. Vu and Laurent Lefèvre. Finite rank distributed control for the resistive diffusion equation using damping assignment. Evolution Equations and Control Theory (EECT) - AIMS, 4(2):205-232, June 2015.

[23] Trang.N.M. Vu and L.Lefèvre. Material balance and closure equations for plasmas in tokamaks. IFAC Workshop on Thermodynamic Foundations of Mathematical Systems Theory, July 13-16 2013.

[24] J. Wesson. Tokamaks. Third edition. Oxford Science Publications, 2004.

[25] H. Zwart, Y. Le Gorrec, B. Maschke, and J. Villegas. Well-posedness and regularity of hyperbolic boundary control systems on a one-dimensional spatial domain. ESAIM: 


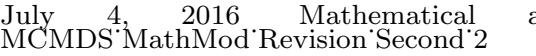

Control, Optimisation and Calculus of Variations, August 2009. 